Research Paper

\title{
Therapeutic Drug Monitoring of Vancomycin in Dermal Interstitial Fluid Using Dissolving Microneedles
}

\author{
Yukako Ito ${ }^{1}$, Yuto Inagaki ${ }^{1}$, Shinji Kobuchi ${ }^{1}, K$ Kanji Takada ${ }^{2}$, and Toshiyuki Sakaeda ${ }^{1, \bowtie}$ \\ 1. Department of Pharmacokinetics, Kyoto Pharmaceutical University, Yamashina-ku, Kyoto, 607-8412, Japan \\ 2. BioSerenTach Inc., Shimogyo-ku, Kyoto, 600-8040, Japan \\ $\triangle$ Corresponding author: Toshiyuki Sakaeda, Ph.D., Department of Pharmacokinetics, Kyoto Pharmaceutical University, Yamashina-ku, Kyoto, 607-8412, \\ Japan. Tel.: +81-75-595-4626; Fax: +81-75-595-6311; Email: sakaedat@mb.kyoto-phu.ac.jp \\ (C) Ivyspring International Publisher. Reproduction is permitted for personal, noncommercial use, provided that the article is in whole, unmodified, and properly cited. See \\ http://ivyspring.com/terms for terms and conditions.
}

Received: 2015.08.19; Accepted: 2016.02.24; Published: 2016.03.16

\begin{abstract}
Objective: To design an alternative painless method for vancomycin (VCM) monitoring by withdrawing interstitial fluid (ISF) the skin using dissolving microneedles (DMNs) and possibly replace the conventional clinical blood sampling method.

Methods: Male Wistar rats were anesthetized with $50 \mathrm{mg} / \mathrm{kg}$ sodium pentobarbital. Vancomycin at $5 \mathrm{mg} / \mathrm{mL}$ in saline was intravenously administered via the jugular vein. ISF was collected from a formed pore at 15, 30, 45, 60, 75, 90, and $120 \mathrm{~min}$ after the DMNs was removed from the skin. In addition, $0.3 \mathrm{~mL}$ blood samples were collected from the left femoral vein.

Results: The correlation between the plasma and ISF VCM concentrations was significantly strong $(r=0.676, p<0.05)$. Microscopic observation of the skin after application of the DMNs demonstrated their safety as a device for sampling ISF.

Conclusion: A novel monitoring method for VCM was developed to painlessly determine concentrations in the ISF as opposed to blood sampling.
\end{abstract}

Key words: vancomycin, dissolving microneedles, interstitial fluid, skin, rats, TDM

\section{Introduction}

Vancomycin is a glycopeptide that has a role in the treatment of Gram-positive methicillin-resistant Staphylococcus aureus infections (MRSA) ${ }^{1}$. Because early use of vancomycin has been associated with a number of adverse effects, including nephrotoxicity, infusion-related toxicities, and possible ototoxicity, therapeutic drug monitoring of vancomycin is advocated. To achieve this, patients are required to visit medical facilities where they are subjected to the painful invasive procedures involving conventional methods of blood sampling from their fingers by using needles. Furthermore, painful blood sampling is also required for blood glucose level measurements when using enzyme assay methods. In addition, concerns regarding infectious diseases often reduce the frequency of blood sampling by patients. Therefore, the development of a painless method for drug monitoring is highly desired. Transcutaneous spectro- scopic methods have been reported previously2, 3; however, electromagnetic energy is almost entirely absorbed by the skin tissue. Therefore, the reliability of these electronic technologies cannot be guaranteed. Although ultrasound ${ }^{4}$, reverse iontophoresis ${ }^{5}$, and electroporation ${ }^{6}$ have also been examined as possible noninvasive methods to monitor glucose levels, they have not yet been clinically introduced because of skin damage, pain, and low accuracy.

Dissolving microneedles (DMNs) have been designed as a transcutaneous drug administration device, and this technology is attracting attention as an alternative method for noninvasive glucose monitoring7, 8 . Microneedles (MNs) were originally designed to percutaneously deliver drugs into the systemic circulation and have been classified into four categories $^{9,10}$. These include (i) hollow type MNs, extremely small needles through, which drug solutions are in- 
jected into the skin; (ii) coating type MNs, made of metallic or silastic substances or both, which are surface-coated with drugs; (iii) pierce type MNs, made of metallic or silastic material or both, which are used to create microconduits in the skin followed by the application of drug solutions, creams, or both after removal of the MNs; and (iv) dissolving microneedles (DMNs), made of soluble polymers such as sodium chondroitin sulfate, dextran, and sodium hyaluronic acid, which are used as a base for the formulation of solid dispersions of drug molecules ${ }^{11,}{ }^{12}$. Of these DMNs, the piercing type, which is made of glass and plastic, has been used for monitoring blood glucose levels. For this process, an MN array is stamped on the skin, and interstitial fluid (ISF) is obtained by applying negative pressure at $200-500 \mathrm{mmHg}^{13}$. Thus, ISF can be collected painlessly using a MN array, and glucose monitoring can be performed with the ISF obtained. However, since the MNs are made of glass and plastic, the risks associated with these non-biological materials must be considered. Despite these concerns, MN technology has been attracting attention as an alternative method for noninvasive therapeutic drug monitoring (TDM) in recent years ${ }^{14}$. MNs are designed to collect dermal interstitial fluid containing biomarkers without the risk or pain associated with or expertise needed for collecting blood.

Microdialysis can be used to collect ISF samples, and the principles have also been previously described ${ }^{15}$. Microdialysis is used to collect ISF from extracellular fluid using the process of diffusion across a semipermeable membrane. Once the probe has been implanted, the tissue analytes diffuse through the membrane from the ISF into the perfusate, and may be sampled and analyzed. Microdialysis requires implantation of the probe in the tissue and, therefore, is difficult in the clinical setting. Furthermore, our DMNs are fabricated from a safe biopolymer, chondroitin sulfate, which has been used in the treatment of arthritis and peripheral circulatory disturbances associated with head injuries. Moreover, DMNs composed of biopolymers as the base material have been investigated for the systemic delivery of peptide/proteins ${ }^{11}, 12$. The high physiological availabilities (PAs) of insulin (91.3-97.7\%) $)^{11}$ and low-molecular-weight heparin (LMWH, 81.5-102.3\%) were previously reported in rats ${ }^{16}$. In addition, a high bioavailability (BA) from the DMNs was shown by recombinant human growth hormone (rhGH, 87.5\%) in rats $^{17}$ and erythropoietin (EPO, 82.1-99.4\%) in mice $^{18}$. The relative BA of interferon (IFN) following a subcutaneous injection of the solution was $79.9-117.8 \%$ in rats ${ }^{19}$. Therefore, DMNs fabricated from these biopolymers are considerably safer than those fabricated from metal and plastic are. In the present study, sodium chondroitin sulfate DMNs were used to collect ISF from rat skin, and then further evaluated as a potential alternative painless method for TDM.

\section{Materials and Methods}

\section{Materials and Animals}

VCM hydrochloride, trifluoroacetic acid (TFA), and sodium chondroitin sulfate were purchased from Wako Pure Chemical Industries Ltd., (Osaka, Japan). Hydroxypropyl cellulose was obtained from Nippon Soda Co., Ltd., (Tokyo, Japan). Male Wistar rats were used in the present study, and standard solid-meal commercial food was obtained from the Japan SLC Inc., (Hamamatsu, Japan). All other chemicals were of reagent grade and used as received.

\section{Preparation of DMN Array Chips}

One milliliter of distilled water was added to 10 mg of sodium chondroitin sulfate and thoroughly mixed to obtain chondroitin glue. The glue was then degassed under reduced pressure and dispensed into a mold containing 300 inverted coneshaped wells with an area of $1.0 \mathrm{~cm}^{2}$. Each well had a depth and surface diameter of 500 and $300 \mu \mathrm{m}$, respectively. The mold was covered with a $300-\mathrm{g}$ steel plate, and the glue was added to the wells and dried. A chip was made of the mixture of cellulose acetate and hydroxypropyl cellulose (10:1) using a Handtab-100 tableting machine (Ichihashi Seiki, Kyoto, Japan). The width and diameter of the chip were 2.0 and $17 \mathrm{~mm}$, respectively. After the plate was removed, glue consisting of $15 \mathrm{mg}$ of chondroitin sulfate and $25 \mathrm{~mL}$ of distilled water was painted over the chip and placed over the mold. After being pressure-dried under a stainless steel plate for $3 \mathrm{~h}$, the chip was removed, and the DMNs were obtained as arrays on the chip.

\section{Preparation of VCM solution administered to rats}

The test solutions of VCM for intravenous (i.v.) and oral administration (p.o.) were prepared by dissolving $250 \mathrm{mg}$ of VCM in $10 \mathrm{~mL}$ of $0.9 \%$ saline and $200 \mathrm{mg}$ in $10 \mathrm{~mL}$ of deionized water, respectively. The i.v and p.o. doses of VCM used in this study were 5.0 and $20.0 \mathrm{mg} / \mathrm{kg}$, respectively.

\section{Collection of ISF using DMNs}

Male Wistar rats (10-week-old) purchased from Nippon SLC Co., Ltd., (SLC, Hamamatsu, Japan) were anesthetized with $50 \mathrm{mg} / \mathrm{kg}$ sodium pentobarbital and their body temperature was maintained at $37^{\circ} \mathrm{C}$ during the experiment by warming with a lamp. The abdominal hair of each rat was shaved (Shaver, Braun Contour 5866, De'Longhi Japan Corp., Tokyo, Japan). 
VCM $(5 \mathrm{mg} / \mathrm{mL})$ in saline was administered i.v. via the jugular vein. At the predetermined time, a DMN array chip was applied to the rat skin using an applicator with a collision speed of $2.0 \mathrm{~m} / \mathrm{s}$ following a second pressure application of $2.5 \mathrm{~N}$ for 1-3 min. After the DMNs had been removed from the rat skin, $2 \mu \mathrm{L}$ of ISF was withdrawn from a formed pore at 15, 30, 45, 60, 75, 90, and 120 min using an Eppendorf pipette and collected into a sample tube. In addition, 0.3-mL blood samples were collected from the left femoral vein. Plasma samples were obtained by centrifuging the blood samples at 12,000 rpm for $15 \mathrm{~min}$ at $4^{\circ} \mathrm{C}$. The ISF and plasma samples obtained were stored at $-80^{\circ} \mathrm{C}$ until used in the assay.

\section{Analytical Methods for VCM}

The quantification assays for VCM in plasma and ISF were performed according to a previously reported method ${ }^{20}$ with some modifications. Briefly, standard samples were prepared by adding aliquots of VCM stock solutions to a drug-free matrix. To determine the levels of VCM, $2 \mu \mathrm{L}$ of ISF was diluted with $98 \mu \mathrm{L}$ of distilled water. Then, exactly $300 \mu \mathrm{L}$ of trifluoroacetic acid/methanol $(2: 1, \mathrm{v} / \mathrm{v})$ was added to a $100-\mu \mathrm{L}$ aliquot of a plasma and ISF sample. The mixture was vortexed for $30 \mathrm{~s}$ and then centrifuged for $15 \mathrm{~min}$ at $12000 \times \mathrm{g}$. The supernatant was diluted with $300 \mu \mathrm{L}$ of distilled water and then transferred to vials. A $30-\mu \mathrm{L}$ sample was injected into the liquid chromatography-tandem mass spectrometry (LC-MS/MS) system. The LC-MS/MS system consisted of an API 3200 triple quadrupole mass spectrometer equipped with a turbo ion spray sample inlet as an interface for electrospray ionization (ESI), an Analyst Workstation (Applied Biosystems, CA, USA), an LC-10AD micropump (Shimadzu Corp., Kyoto, Japan), and an AS8020 automatic sample injector (Toso, Tokyo, Japan). The mobile phase was distilled water-acetonitrile $(9: 1, \mathrm{v} / \mathrm{v})$ containing $0.1 \%$ acetic acid at a flow rate of $0.2 \mathrm{~mL} / \mathrm{min}$. The analytical column was a Quicksorb ODS (2.1 mm i.d. $\times 150 \mathrm{~mm}$, $5 \mu \mathrm{m}$ size, Chemco Scientific Co., Ltd., Osaka, Japan) maintained at $50^{\circ} \mathrm{C}$. Ionization was performed via the turbo ion spray inlet in the positive ion mode. The ion spray voltage and temperature were set at $5500 \mathrm{~V}$ and $500^{\circ} \mathrm{C}$, respectively. Nitrogen gas was used for instrument operation, and the values for gas 1 , gas 2 , curtain gas, and collision gas were set at 50,70, 10, and 2 , respectively. The declustering potential (DP), entrance potential (EP), collision energy (CE), and collision cell exit potential (CXP) were set at 28, 7.5, 21, and $1.6 \mathrm{~V}$, respectively. Multiple reaction-monitoring (MRM) analyses were performed using transitions at $\mathrm{m} / \mathrm{z} 725.5 \rightarrow 144.0$. The lower limits of detection and the limits of quantification for VCM were less than 10 $\mathrm{ng} / \mathrm{mL}$ for the $100-\mu \mathrm{L}$ samples of each matrix. The standard curves were linear over the lower limits of quantification $(r>0.99)$.

\section{Microscopy of Rat Skin}

Male Wistar rats weighing $315 \pm 13 \mathrm{~g}$ were anesthetized with $50 \mathrm{mg} / \mathrm{kg}$ sodium pentobarbital and their body temperature was maintained at $37^{\circ} \mathrm{C}$ during the experiment by warming. The hair on the abdominal region of each rat was shaved, and an image of the skin was captured using a Nikon D-200 camera (Nikon, Tokyo, Japan) under normal light. Then, a DMN array chip was administered to the rat skin and before its removal from the abdominal skin, the image of the skin was also captured using the camera. In addition, $1 \mathrm{~h}$ after the removal of the DMN array chip, the skin image was also captured.

All animal protocols were approved by the Institutional Animal Care and Use Committee. Experiments were conducted in accordance with the Guidelines for Animal Experimentation, of the Kyoto Pharmaceutical University.

\section{Statistical Analysis}

All values are expressed as the mean \pm standard error (SE). Differences were assumed significant at $p<$ 0.05 (Student's unpaired $t$-test). The coefficient of determination (R2) was evaluated using the Pearson's correlation coefficient test at $\mathrm{p}<0.05$.

\section{Results and Discussion}

DMNs composed of chondroitin sulfate prior to application to the rat skin are shown in the upper panel in Fig. 1. The chip included 225 DMNs on an area of $1.0 \mathrm{~cm}^{2}$. The middle panel of Fig. 1 shows an array of DMNs while and the lower panel illustrates a magnification of one DMN from the middle panel. The mean length and basement diameter of the DMNs was $501.8 \pm 2.1$ and $274.6 \pm 1.6 \mu \mathrm{m}$.

To ascertain the safety of DMNs as a device for sampling ISF, the rat skin was examined pathologically, as shown in Fig. 2. The upper right and lower left panels illustrate the rat skin immediately, and $5 \mathrm{~min}$ after insertion of DMNs, respectively, and the microconduits were made on the skin by the application of the DMNs. The lower right panel in Fig. 2 presents the rat skin condition $10 \mathrm{~min}$ after insertion of DMNs. Microconduits were not detected, and the rat skin condition recovered to normal. These results indicate that no damage was caused by the inflammation from the DMN application. These results were supported by our previous studies, which showed no evident inflammation and microscopic destruction of skin tissue in the microscopic image data and recovery of the rat skin after 
insertion of the DMNs ${ }^{21}, 22$. Therefore, this device could be a noninvasive sampling method.
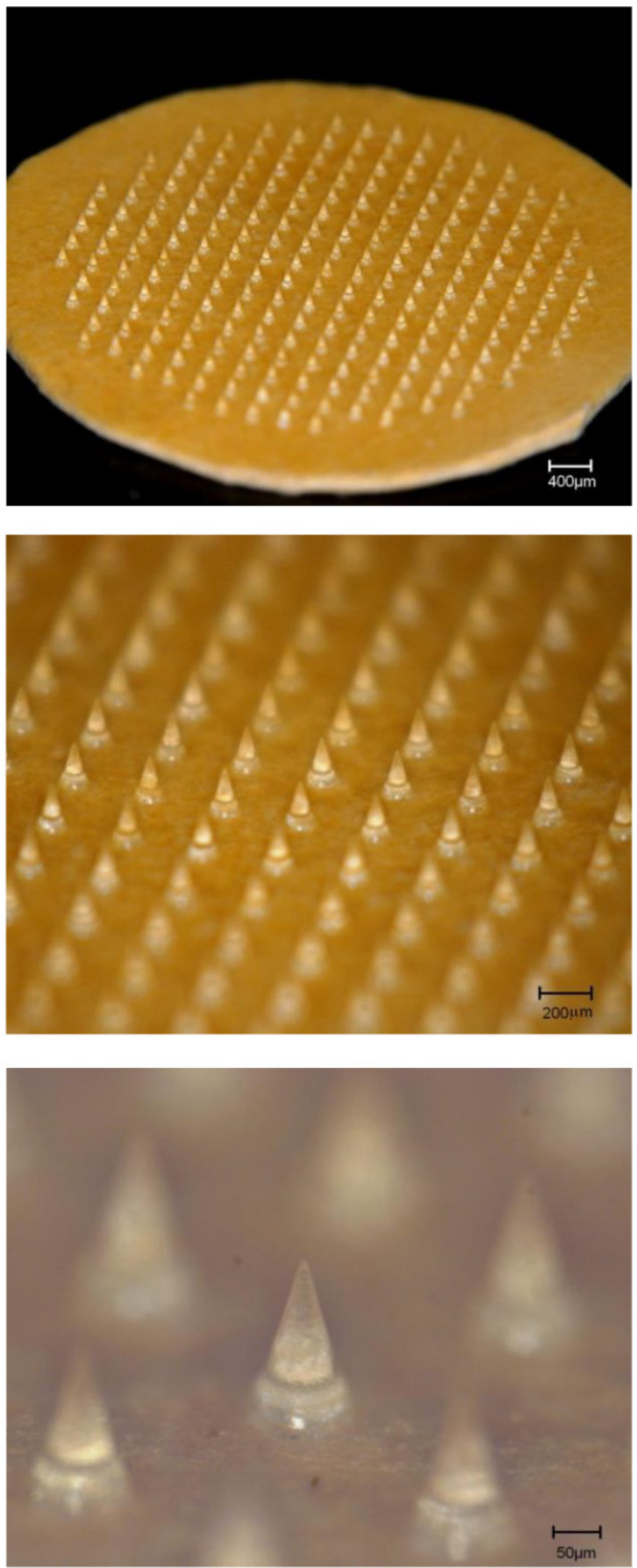

Fig. 1. Dissolving microneedles (DMNs) made of chondroitin sulfate prior to application on rat skin. A total of 225 DMNs arrays were formed on a $1.0 \mathrm{~cm}^{2}$ chip. Mean length and basement diameter of the DMNs were $501.8 \pm 2.1$ and $274.6 \pm 1.6 \mu \mathrm{m}$

Next, the concentrations of VCM in the plasma and ISF were compared, and their pharmacokinetic profiles are shown in Fig. 3. The mean concentration of VCM in plasma was higher than that in ISF was 30 min after administration because the distribution process of VCM was not completed in the early phase. Furthermore, the mean concentration-time curve of ISF was reversed after $30 \mathrm{~min}$ and the concentrations of VCM in plasma and ISF declined similarly. The concentration of VCM in the ISF remained higher than that in plasma, suggesting that the distribution attained steady state and the transition of VCM from the tissue back to plasma was not completed. Therefore, the tissue affinity of VCM appeared to be stronger than that of the plasma.
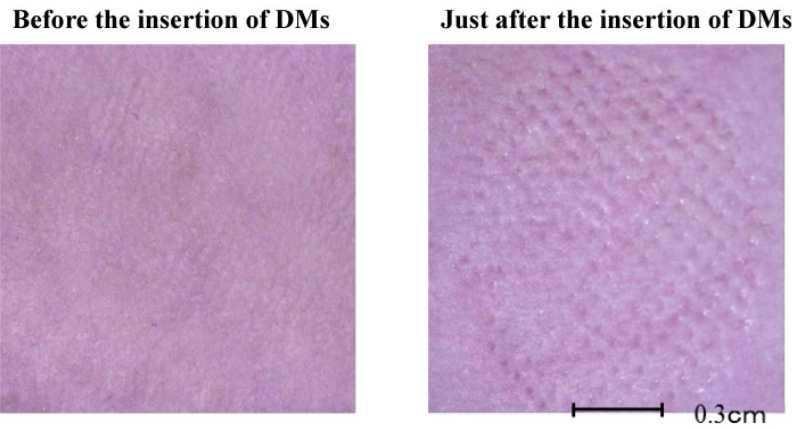

5 min after the insertion of DMs
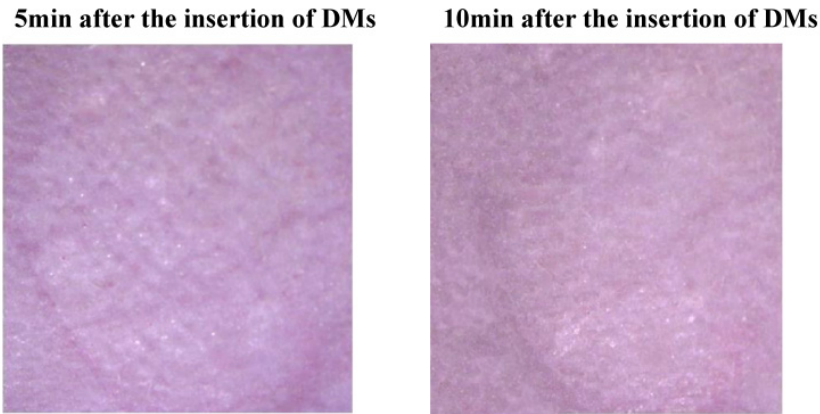

Fig. 2. Pathological examination skin after percutaneous application of dissolving microneedles (DMNs). Upper right panel shows skin condition immediately after insertion of DMNs. Pressure application of DMNs created pores on skin. Lower left panel shows skin condition $5 \mathrm{~min}$ after insertion of DMNs with pores still evident and not recovered. Lower right panel shows skin condition $10 \mathrm{~min}$ after insertion of DMNs. Pores disappeared, and skin structure recovered to normal physiology.

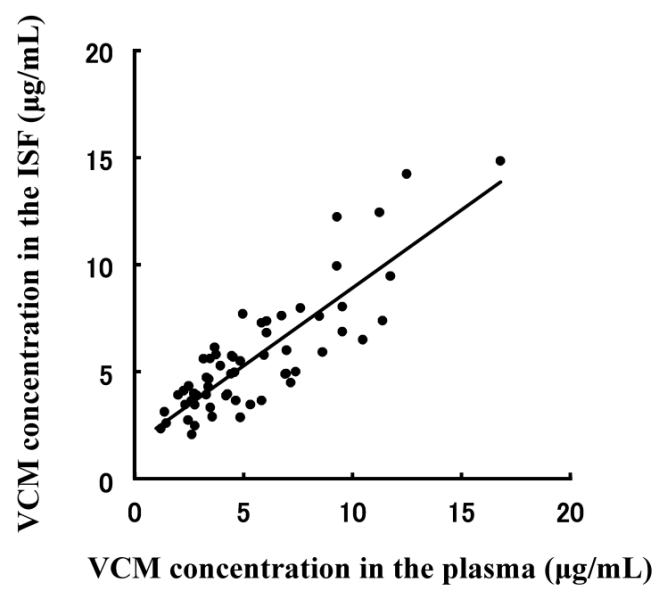

Fig. 3. Correlation between plasma and interstitial fluid (ISF) vancomycin (VCM) concentrations. A strong correlation $(r=0.676, p<0.05$, Pearson's correlation coefficient test) was observed between plasma and ISF VCM concentrations. 
To evaluate the efficiency of the DMN TDM using ISF sampling as a noninvasive method, the correlation of the VCM concentration in plasma and ISF following administration to rats was plotted in Fig. 4. A good correlations $(r=0.717, p<0.05$, Pearson's correlation coefficient test) was observed between both matrices. However, the values at higher concentration were not correlated, and this may be because they were observed prior to the steady state distribution of VCM. After the distribution had attained steady state, the correlation coefficient value obtained would be higher than it was before. These results suggest that this method may be a suitable method for TDM instead of blood sampling.

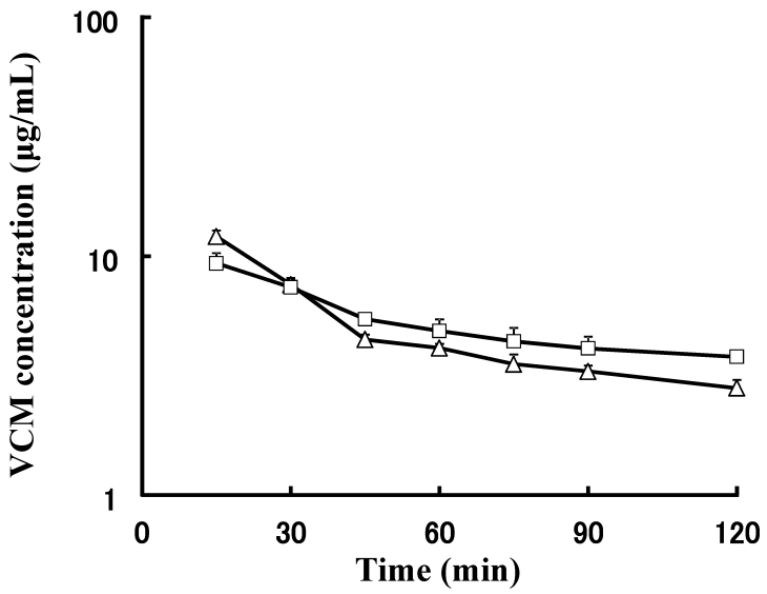

Fig. 4. Mean plasma and interstitial fluid (ISF) vancomycin (VCM) concentration-time curves of rats administered intravenous (i.v.) bolus injection. Concentration-time curves for VCM in both matrices were similar and showed parallel decline.

TDM requires frequent blood drawing, which may be a challenge in neonates, infants, children, or the elderly ${ }^{23}$. Reduction in compliance is widespread because drawing blood increases patient discomfort and inconvenience.

Kiang et al. ${ }^{24}$ reported an extensive list of clinically used contemporary drugs that are subject to TDM, and the feasibility of sampling and obtaining reliable drug concentrations in ISF collected using ultrafiltration probes in rabbits. VCM showed comparable exposure and similar concentration-time curves in both ISF and blood in rabbits. They found that ISF could replace the blood sampling because there was a linear elimination and reliable correlation between the concentrations and area under the concentration-time curve (AUC). These previous reports are supported by our present results. We obtained a good correlation between the concentrations of VCM in the ISF and plasma. Moreover, the delay in VCM concentration increase in the ISF in the previous study was similar to what we observed, which may be be- cause the distribution of VCM from plasma to tissue has a lag time prior to completion.

Hauschild et al. ${ }^{25}$ also compared the concentrations of pradofloxacin in the ISF and plasma in dogs. They also used the ultrafiltration probe for collecting the ISF, and a delay was observed in the increase in paradofloxacin concentration in the ISF, although it was administered orally to dogs. Furthermore, the concentration of VCM in the ISF was determined immediately after administration. They described $\mathrm{VCM}$ as the only drug evaluated that could be categorized as suitable for TDM in the ISF because of comparable exposures and similar concentration-time profiles in both ISF and blood. In their model $(n=4)$, VCM exhibited reduced maximum plasma drug concentration (Cmax) in the ISF $(32.1 \pm 2.6 \mu \mathrm{g} / \mathrm{mL})$ compared with the blood $(80.2 \pm 18.5 \mu \mathrm{g} / \mathrm{mL}, \mathrm{p}<$ 0.05); however, they obtained similar AUCs for the two matrices (ISF vs. blood, $75.3 \pm 7.7$ vs. $89.8 \pm 15.7$ $\mu \mathrm{g} \mathrm{h} / \mathrm{mL}$, respectively). A delayed average time to achieve maximum plasma drug concentration (Tmax) of $0.66 \mathrm{~h}$ was observed with ISF. The concentration-time profiles for VCM in both matrices were similar, and this is evidenced by the parallel decline in their terminal elimination phases in log-transformed plots.

Those reports described the use of microdialysis in the collection of the ISF, which is not suitable for use in humans because implantation is required and the patients have to bear the burden of the pain, inconvenience, and risk of developing infections. Therefore, the DMN was designed as a device for more convenient invasive patient monitoring.

Donnely et al. ${ }^{26}$ reported on MN-mediated minimally invasive patient monitoring. The development of the MN-based monitoring would be focused on further improving its utilities to enhance the ease-of-use for patients and clinicians. The worldwide trend is to improve the health-related quality of life for patients by decreasing the risks of developing infections and frequency of inconvenient monitoring using invasive blood sampling. El-Laboudi et al. ${ }^{27}$ also reported continuous glucose monitoring using the MN array device and focused on the mechanism of insertion and safety profile such as the small risk of infection, painless sample collection, no bleeding, and rapid skin recovery. Romanyuk et al..$^{23}$ investigated the method of collection of analytes from the MN patches. They prepared hydrogel MNs with poly(methyl vinyl ether-alt-maleic acid) and poly(ethylene glycol). The analytes in the ISF from the hydrogel MN could be easily transferred to the microtubes or multiwell plates in the laboratory for analysis. Therefore, their analytes were detected outside the hydrogel MN. However, when the volume of 
the ISF is limited, a highly sensitive analytical method is needed. On the other hand, our method of sample collection involves withdrawing the ISF from the microconduit by inserting the DMN. Therefore, the volume of the ISF obtained was sufficient for the determination of the drug concentration.

\section{Conclusion}

Herein, a method of monitoring VCM in the ISF by using DMNs was investigated as an alternative to blood sampling. The pharmacokinetic profiles of VCM in the ISF and plasma were compared, and drug concentrations showed a similar decline except at 30 min after administration. The correlation of the concentrations of VCM in the ISF and plasma was significantly positive. Moreover, microscopic observation revealed that the integrity of the skin was maintained, which suggests that the DMN array chip is a safe device for ISF sampling. Therefore, drug monitoring in ISF using the DMN array chip has the potential to replace blood sampling in VCM TDM.

\section{Acknowledgments}

This study was supported by a grant from the Ministry of Education, Culture, Sports, Science, and Technology (MEXT)-Supported Program for the Strategic Research Foundation at Private Universities, 2008-2013. This study was also supported by a Grant-in-Aid for scientific research provided by MEXT, 2010-2013.

\section{Competing Interests}

The authors have declared that no competing interest exists.

\section{References}

1. Kiang TKL, Häfeli UO, Ensom MHH. A comprehensive review on the pharmacokinetics of antibiotics in interstitial fluid spaces in humans: Implication on dosing and clinical pharmacokinetic monitoring. Clinical Pharmacokinetics 2014; 53: 695-730.

2. Kajiwara K, Fukushima H, Kishikawa H, Nishida K, Hashiguchi $Y$, Sakakida M, Uehara M, Shichiri M. Spectroscopic quantitative analysis of blood glucose by Fourier transform infrared spectroscopy with an attenuated total reflection prism. Medical Progress Through Technology 1992; 18: 181-189.

3. Yamakoshi K, Yamakoshi I. Pulse glucometry. A new approach for noninvasive blood glucose measurement using instantaneous differential near-infrared spectrophotometry. Journal of Biomedical Optics 2006; 11: 054028.

4. Lee S, Nayak V, Dodds J, Pishko M, Smith NB. Glucose measurements with sensors and ultrasound. Ultrasound in Medicine and Biology 2005; 31: 971-977.

5. Sieg A, Guy RH, Delgado-Charrro MB. Noninvasive glucose monitoring by reverse iontophoresis in vivo: application of the internal standard concept. Clinical Chemistry 2004; 50: 1383-1390.

6. Murthy SS, Kiran VSR, Mathur SK, Murthy SN. Noninvasive transcutaneous sampling of glucose by electroporation. Journal of Diabetes Science and Technology 2008; 2: 250-254.

7. Prausnitz RM. Microneedles for transdermal drug delivery. Advanced Drug Delivery Reviews 2004; 56: 581-587.

8. Bariya SH, Gohel MC, Mehta TA, Sharma OP. Microneedles: an emerging transdermal drug delivery system. The Journal of Pharmacy and Pharmacology 2012; 64: 11-29.

9. Coulman SA, Anstey A, Gateley C, Morrissey A, McLoughlin P, Allender C, Birchall JC. Microneedle mediated delivery of nanoparticles into human skin. International Journal of Pharmaceutics 2009; 366: 190-200.
10. Kim YC, Park JH, Prausnitz MR. Microneedles for drug and vaccine delivery. Advanced Drug Delivery Reviews 2012; 64: 1547-1568.

11. Ito Y, Hagiwara E, Saeki A, Sugioka N, Takada K. Feasibility of microneedles for percutaneous absorption of insulin. European Journal of Pharmaceutical Sciences 2006; 29: 82-88.

12. Takada K. Microfabrication derived DDS: From batch to individual production. Drug Discovery and Therapeutics 2008; 2: 140-155.

13. Wang PM, Cornwell M, Prausnitz MR. Minimally invasive extraction of dermal interstitial fluid for glucose monitoring using microneedles. Diabetes Technology and Therapeutics 2005; 7: 131-141.

14. Ito Y, Taniguchi M, Hayashi A, Anai M, Morita S, Ko E, Yoshimoto N, Yoshii Y, Kobuchi S, Sakaeda T, Takada K. Application of dissolving microneedles to glucose monitoring through dermal interstitial fluid. Biological and Pharmaceutical Bulletin 2014; 37: 1776-1781.

15. de la Peña A, Liu P, Derendorf H. Microdialysis in peripheral tissues. Advanced Drug Delivery Reviews 2000; 45: 189-216.

16. Ito $\mathrm{Y}$, Murakami A, Maeda $\mathrm{T}$, Sugioka $\mathrm{N}$, Takada K. Evaluation of self-dissolving needles containing low molecular weight heparin (LMWH) in rats. International Journal of Pharmaceutics 2008; 349: 124-129.

17. Ito $Y$, Ohashi $Y$, Shiroyama K, Sugioka N, Takada K. Self-dissolving micropiles for the percutaneous absorption of human growth hormone in rats. Biological and Pharmaceutical Bulletin 2008; 31: 1631-1633.

18. Ito Y, Yoshimitsu J, Shiroyama K, Sugioka N, Takada K. Self-dissolving microneedles for the percutaneous absorption of EPO in mice. Journal of Drug Targeting 2006; 14: 255-262.

19. Ito Y, Saeki A, Shiroyama K, Sugioka N, Takada K. Percutaneous absorption of interferon- $\beta$ by self-dissolving micropiles. Journal of Drug Targeting 2008; 16 : 243-249.

20. Shibata N, Ishida M, Prasad YV, Gao W, Yoshikawa Y, Takada K. Highly sensitive quantification of vancomycin in plasma samples using liquid chromatography-tandem mass spectrometry and oral bioavailability in rats. Journal of Chromatography B: Analytical Technologies in the Biomedical Life Sciences. 2003; 789: 211-8.

21. Ikejiri A, Ito Y, Naito S, Takada K. Two- and three-layered dissolving microneedles for transcutaneous delivery of model vaccine antigen in rats. Journal of Biomaterials and Nanobiotechnology, 2012; 3: 325-334.

22. Ito $Y$, Hamazaki N, Higashino H, Miyamoto N, Murakami $Y$, Inoue N, Takada $\mathrm{K}$. Recovery rate of rat skin micropores made by dissolving microneedles. Journal of Drug Delivery Science Technology 2013; 23: 577-581.

23. Romanyuk AV, Zvexdin VN, Samant P, Grender MI, Zemlyanova M, Prausnitz MR. Collection of analytes from microneedle patches. Analytical Chemistry 2014; 86: 10520-10523.

24. Kiang TKL, Schmitt V, Ensom MHH, Chua B, Häfeli UO. Therapeutic drug monitoring in interstitial fluid: A feasibility study using a comprehensive panel of drugs. Journal of Pharmaceutical Sciences 2012; 101: 4642-4652.

25. Hauschild G, Rohn K, Engelhardt E, Sager M, Hardes J, Gosheger G. Pharmacokinetic study on pradofloxacin in the dog - comparison of serum analysis, ultrafiltration and tissue sampling after oral administration. BMC Veterinary Research 2013; 9: 32-39.

26. Donnely RF, Mooney K, Caffarel-Salvador E, et al. Microneedle-mediated minimally invasive patient monitoring. Therapeutic Drug Monitoring 2014; 36: $10-17$.

27. El-Laboudi, A, Oliver NS, Cass A, Johnston D. Use of microneedle array devices for continuous glucose monitoring: a review. Diabetes Technology and Therapeutics 2013; 15: 101-115. 